\title{
Fog and dense gas disrupt mosquito flight due to increased aerodynamic drag on halteres
}

\author{
Andrew K. Dickerson ${ }^{1}$, Peter G. Shankles ${ }^{1}$, Bruce E. Berry Jr. ${ }^{1}$, David L. Hu ${ }^{1,2, *}$ \\ Schools of Mechanical Engineering ${ }^{1}$, and Biology ${ }^{2}$ \\ Georgia Institute of Technology \\ * Corresponding author: hu@me.gatech.edu
}

April 1, 2015

\begin{abstract}
Chemical insecticide foggers have long been used to control mosquito populations and reduce the spread of malaria, West Nile virus, and dengue fever. In this study, we show simple fogs of water droplets, devoid of chemicals, are effective at obstructing the flight of Anopheles freeborni mosquitoes. Using high-speed video, we film the flight of mosquitoes in denser-than-air fluids such as fogs produced by household humidifiers and heavy gas. In gases with more than twice the density of air, mosquitoes cannot maintain flight stability, but pitch and roll uncontrollably until they fall to the ground. We here show the increased drag of denser-than-air flight environments is sufficient to render a mosquito's gyroscopic sensors and wingbeat controllers, their halteres, ineffective. Anomalous drag forces are estimated with steady and unsteady drag analyses. We glue masses of 10 nanograms to each haltere, equivalent to $0.0005 \%$ the mass of the mosquito. Mosquitoes with such a minor treatment executed flight patterns similar to mosquitoes in fog, indicating a mosquito's halteres are highly tuned to aerodynamic properties of normal air, making them sensitive to contaminants. The failure of mosquito flight in fog reveals potential new methods for mosquito control that do not require insecticide-laced particles.
\end{abstract}

Keywords: fogs, insect-flight sensing, free vibration, Coriolis forces

\section{Introduction}

Half of the world's population resides in regions where malaria transmission is a risk ${ }^{1}$. In 2010 , approximately 216 million clinical malaria episodes resulted in an estimated 655,000 deaths. Transmission of malaria to humans occurs through the bite of an infected female mosquito ${ }^{2}$. Mosquito control methods include intermittent preventative treatment such as removal of stagnant water pools ${ }^{3}$ and usage of insecticide-treated bed nets ${ }^{4}$. Indoor residual spraying ${ }^{5}$, chemical larviciding ${ }^{6}$, and insecticide spraying ${ }^{7}$ aim to kill adult mosquitoes. The latter technique works by depositing toxins on the insect body, through encounters with airborne particles or insecticide-ridden surfaces. Toxins are carried by water or oil micro-droplets. While these insecticides possess acceptably low toxicity to humans, strict regulations govern their use and application. Spraying and fogging should be done at the peak of adult mosquito activity, which is highly variable among species and requires repetition, which can be expensive. By studying the vulnerabilities of mosquito flight, unforeseen strategies for mosquito mitigation free of insecticides may be found. The chosen model organism in the study is the Anopheles freeborni mosquito, an established malaria vector.

In this study, we investigate mosquito flight vulnerabilities by probing the limits of their locomotion through particulate environments. Many insects, including mosquitoes, thrive in wet, humid locations such as the tropics ${ }^{8}$. Abundant moisture often brings about heavy rains and dense fogs, through which mosquitoes must fly in search of food and mates. Introducing particles such 
as raindrops, into the flight medium, increases the cost of flight performance for bats and birds ${ }^{9}$ Previous work has shown mosquitoes can survive rain showers ${ }^{10}$ by virtue of their low mass which greatly reduces the force of impact. A mosquito's flight performance through dense fog is poorly understood, despite the widespread use of insecticide foggers. Little is known of how small particles can affect the insect's air-wing interactions and sensing of its environments.

Airborne particles can broadly be characterized into one of two categories, fog and haze. Fog is defined as providing less than $1 \mathrm{~km}$ of visibility ${ }^{11}$. Depending on the altitude ${ }^{12}$, fog naturally consists of small water droplets, in the liquid or frozen state, of size $1-10 \mu \mathrm{m}$ in diameter and concentrations $25-100 \mathrm{mg} / \mathrm{m}^{3}$. Fog has a bimodal distribution; the larger fog particles close to the ground have an average diameter of $5 \mu \mathrm{m}$, whereas the higher altitude particles have a diameter of $0.3-0.6 \mu \mathrm{m}$. On the other hand, haze consists of $0.2 \mu \mathrm{m}$ dust and smog particles, with little vertical variation. Fog and haze particle size can be measured much like insecticide particle size, through light scattering and holographic techniques ${ }^{13,14}$. In comparison to natural fogs, insecticides involve drop sizes comparable to that of natural fogs, but of one millionth the density, less than $100 \mathrm{ng} / \mathrm{m}^{3}$ when dispersed, rendering them invisible. ${ }^{15}$. However, during dispersion, fogs and sprays at output nozzles are very dense and visible.

Dense fog has been known to disrupt avian vision and navigation ability ${ }^{16}$. Like birds, insects also use a combination of visual and force-mediated feedback to navigate ${ }^{17}$. Tethered fruitflies and houseflies use an optomotor response to adjust their thrust and torque for visual cues positioned in front of their eyes ${ }^{18}$. Insects without gyroscopes, like the desert locust, employ other means of flight sensing ${ }^{19,20}$ such as sensitive hairs on their hind limbs to detect wind direction. The small tortoiseshell butterfly uses its antenna to detect flight speed ${ }^{21,22}$, while the hawkmoth vibrates its large antennae to detect Coriolis forces ${ }^{23}$. Only recently has any work been done on how particles affect olfactory sensors on cockroaches ${ }^{24}$, but it well-established that mosquitoes rely heavily on olfactory cues to find food and mates ${ }^{25}$. However, the effect of airborne particles on these sensing systems has not been systematically studied, and sensing systems are very species specific.

In this study, we focus on flight sensing due to the interaction between fog and a mosquito's halteres. Halteres, small knobbed structures evolved from hind wings, flap with the same frequency as wings and serve several in-flight functions ${ }^{26-28}$ (Fig.1a,b). Halteres, provide proprioceptive feedback by detecting Coriolis forces and play a crucial role in the neuromuscular circuit generating the wingbeat rhythm in Dipterans ${ }^{17,29}$. By activating the motor neurons of minuscule steering muscles, halteres help regulate wing motion through a reflexive feedback loop with these muscles. Haltere-bearing insects have irregular fields of campaniform sensilla embedded in their cuticle at the base of the haltere shaft ${ }^{30}$. The architecture of the campaniform structures gives them the ability to detect small strains in the cuticle as the haltere flaps. Historically, halteres have been thought to sense inertial, centrifugal, and gravitational forces in addition to Coriolis forces ${ }^{27}$.

Mosquito halteres have not been the focus of much investigation, but the function and dynamics of fruitfly halteres has been well studied. In tethered fruitflies, adding mass to one haltere using glue acts to reduce peak velocity and amplitude of body saccades ${ }^{17}$. Ablating one fruitfly haltere has the opposite effect by accelerating in-flight maneuvers. In some situations, haltere augmentation results in the adoption of a flapping frequency different from that of the wings ${ }^{31,32}$. Sensory feedback from the haltere is read by the wing control mechanisms on a stroke-by-stroke basis, even in steady flight ${ }^{33,34}$, so the effects of haltere augmentation in fruitflies may be due to the disruption in synchronous wing-haltere signals ${ }^{17}$. Houseflies with halteres removed show a complete lack of control over flight ${ }^{26}$.

In this study we investigate the ability of Anopheles freeborni mosquitoes to maintain flight in hyperdense air. In $\S 2$, we present our measurements of the likelihood and promptness of flight failure in heavy air, and a calculation of the aerodynamic forces involved. To show such forces are sufficient 
to perturb halteres, we laden halteres with additional mass. In $\S 3$, we present a discussion of our results and avenues for future work. Our experimental methods for insect handling and imaging are provided in $\S 4$, and we summarize our main points in $\S 5$.

\section{Results}

\subsection{Flight failure in fog and heavy gas}

We performed hundreds of experiments with Anopheles freeborni flying in both fog and 38 mixtures of gas with various densities. Fog here refers to the output from a commercial humidifier, consisting of $5-35 \mu \mathrm{m}$ diameter water droplets, with an average diameter of $15 \mu \mathrm{m}$. The microscopic droplets increase the aggregate fluid density through which a mosquito flaps. We define flight failure as a rapid descent to the ground and the subsequent inability to resume flight. Failure is observed when a mosquito encounters a stream laden with fog particles or when introduced to a quiescent medium with suspended fog particles. Landing on the floor of a container is atypical behavior, as mosquitoes prefer to land on walls and ceilings, regardless of the species ${ }^{35}$. We observe less than $10 \%$ of individuals from a group (number of trials, $\mathrm{N}=20$ ) of Anopheles freeborni choose to rest on the floor of their container under normal conditions.

Every case of flight failure ( $\mathrm{N}>100$ ) involves pitching (Fig.1c, Supp. Video 1) or rolling (Fig.1d, Supp. Video 2), generating inversion or steep angles of orientation with respect to the horizontal flight position. When mosquitoes have rotated $90^{\circ}$ relative to vertical, we surmise the lift generated by wings cannot maintain their position and they quickly begin falling downward. In other instances, the mosquito maintains its typical orientation and even generates lift in fog, or heavy gas, for fractions of a second, as shown by the upward flight in Supp. Videos 1 \& 2. Nevertheless, these periods of normal flight, which occur in approximately $10 \%$ of the flights filmed, are so sporadic and unstable that mosquitoes inevitably fall to the floor within seconds of introduction of heavy fog. Fog particle deposition on the wings or body is not the cause of flight instability, as we observe flight may resume immediately after fog particles are removed from the air, and that a mosquito blanketed with small drops on its wings and legs retains the ability to fly in dry air.

Mosquitoes behave similarly in both fog and heavy gas, indicating the increased aggregate density of the medium is the cause of flight failure, rather than the particulate nature of fog. A heavy gas with the same average density as fog causes nearly identical flight failure, as shown in Supp. Video 3. Heavy gas is also transparent, indicating that the opacity of the medium and occlusion of vision is not a factor in their flight failure.

In Fig.2a,b we present flight tracks for mosquitoes in normal air and hyperdense air, respectively. As demonstrated by the flight track in Fig.2a, a mosquito in normal air can remain aloft for more than 4 seconds before landing . Conversely, in hyperdense air, the descent behavior is clearly distinct. Fig.2b shows the mosquito falls quickly from the ceiling to the floor of the container. The mosquito falling in the most dense gas (red) shows the least control over flight, falling at an average falling speed of $27 \mathrm{~cm} / \mathrm{s}$ over $0.55 \mathrm{~s}$.

We measured the flight time before grounding when the mosquito is dropped from a height $H=15 \mathrm{~cm}$, in air made heavier by the addition of R134a. Humidifier fog was not employed in this experiment due to its propensity for settling and aggregating on container walls. Fig.3a shows descent time becomes both shorter and more predictable with increasing gas density. The fastest possible descent time is $T=0.11 \mathrm{~s}$, the time needed to fall a distance $H$ at terminal velocity, 136.5 $\pm 9.5 \mathrm{~cm} / \mathrm{s}(\mathrm{N}=10)$. The shortest descent times observed are twice this minimal time, indicating 
mosquitoes are slowing their descent by attempting flight. During descent, mosquitoes flap their wings at varying frequencies $(150-400 \mathrm{~Hz})$, but continue to fall on their sides or inverted.

Fig.3b shows the relation between gas density and proportion of mosquitoes able to sustain flight for over 3 seconds. Over an intermediate range of gas density, $0.9-1.6 \mathrm{~kg} / \mathrm{m}^{3}$, mosquitoes can stay aloft indefinitely. The corresponding success rate in this range is slightly less than $100 \%$ because of experimental error. In our experiments, we do not control for initial orientation of the mosquito. Thus, a few mosquitoes are released upside-down upon entry into the container and, if unable to recover in less than one second, strike the ground.

At higher gas densities, between 1.6 and $2 \mathrm{~kg} / \mathrm{m}^{3}$, only $50 \%$ mosquitoes can maintain steady flight. At the highest gas densities tested, greater than $2 \mathrm{~kg} / \mathrm{m}^{3}$, no mosquitoes are able to maintain flight. At this threshold, the volume fraction of water in the flight medium is $8 \times 10^{-4}$, and a cubic millimeter contains approximately 450 droplets. To investigate further the mechanism for flight failure, we consider in the next section the aerodynamic forces on the mosquitoes' flapping halteres.

\subsection{Flight failure due to haltere-fluid interaction}

In our previous section, we identified the air densities in which mosquitoes can fly. Mosquitoes fail to sustain flight in humidifier fog, which has a density up to $2 \mathrm{~kg} / \mathrm{m}^{3}$. We hypothesize this inability to fly is caused by the interaction between the mosquito haltere and ambient fluid. Furthermore, after landing, mosquitoes remain grounded until the fog particle concentration falls below $1.7 \mathrm{~kg} / \mathrm{m}^{3}$. Thus, it appears mosquitoes can also sense fog in their surroundings and consequently choose not to take off.

We measure haltere kinematics and geometry using high speed videography and scanning electron microscopy. Halteres are drumstick-shaped, with a characteristic length of $\ell=250 \mu \mathrm{m}$ and radius, at the widest point, of $R=60 \mu \mathrm{m}$, as shown in Fig.1a,b. In comparison to fog droplet of diameter $5-35 \mu \mathrm{m}$, a haltere has $5-1730$ the volume of a fog droplet. When tethered mosquitoes are stimulated, they flap both wings and halteres for brief periods using a full range of motion. We observe haltere flapping is sinusoidal with similar kinematics for all gas densities tested $(0.2-4.2$ $\left.\mathrm{kg} / \mathrm{m}^{3}\right)$. In normal air, wings and halteres flap at a frequency of $f=375 \pm 42 \mathrm{~Hz}(\mathrm{~N}=19)$, on the same order as the flapping frequency in dense fog $\left(\rho=2 \mathrm{~kg} / \mathrm{m}^{3}\right)$ of $f=303 \pm 37 \mathrm{~Hz}(\mathrm{~N}=9)(\mathrm{P}-$ value $<0.0001)$. The $20 \%$ decrease in flapping frequency due to a $67 \%$ increase in medium density suggests that form drag on the wings is constant within both fog and air. Drag force $F_{\mathrm{D}} \sim \rho f^{2}$ and $1.67 \times 0.8^{2} \approx 1$.

In the following calculations, we use a characteristic flapping frequency of $f=300 \mathrm{~Hz}$. The haltere flapping amplitude is $A=97 \pm 2^{\circ}(\mathrm{N}=6)$. The time-dependent, linear velocity of a haltere in simple harmonic motion can be written $V(t)=A \ell \omega \cos (\omega t)$, where $\omega=2 \pi f$. The peak velocity of the haltere-tip is therefore $V_{\max } \approx 2 \pi f A \ell=0.74 \mathrm{~m} / \mathrm{s}$, using a flapping frequency of $f=300 \mathrm{~Hz}$, and flapping amplitude $A=\pi / 2$ radians. At densities where flight failure begins, more than 300 fog particles are contained within $1 \mathrm{~mm}^{3}$ of a mosquito's flight medium. The flapping halteres will encounter upwards of 2,000 of these particles in 1 second of flight, assuming the shaft's frontal area is negligible compared with that of the bulb.

The total force on the flapping haltere in hovering, non-rotational flight consists of a sum of drag force $F_{D}$, and inertial force $F_{i}$,

$$
F_{\text {haltere }}=F_{\mathrm{D}}+F_{\mathrm{i}}
$$


where each term may be expressed

$$
\begin{aligned}
F_{\mathrm{D}, \mathrm{s}} & =\frac{1}{2} C_{\mathrm{D}} \rho \pi R^{2} V^{2}, \\
F_{\mathrm{i}} & =m \frac{\mathrm{d} V}{\mathrm{~d} t} .
\end{aligned}
$$

The haltere has mass $m$, radius $R$ and time-dependent velocity $V$. Dimensionless coefficient $C_{\mathrm{D}}=$ 5.1 , the drag coefficient for a sphere in steady flow. The corresponding Reynolds number $\operatorname{Re}=$ $2 R \rho V_{\max } / \mu=8$, for a medium density of $1.7 \mathrm{~kg} / \mathrm{m}^{3}$, and dynamic viscosity of air $\mu=1.98 \times 10^{-5}$ $\mathrm{kg} / \mathrm{m}-\mathrm{s}$.

For flight medium density $\rho=2.0 \mathrm{~kg} / \mathrm{m}^{3}$ about $67 \%$ greater than that of air $\left(\rho_{\text {air }}=1.2\right.$ $\left.\mathrm{kg} / \mathrm{m}^{3}\right)$, the anomalous peak drag force is $\Delta F_{\mathrm{D}, \mathrm{s}}=\frac{1}{2}\left(\rho-\rho_{\text {air }}\right) C_{\mathrm{D}} \pi R^{2}(A \ell \omega)^{2}=12.4 \mathrm{nN}$, which is applicable only in the case when wings are not pushing air over the halteres. We believe this to be true of hovering mosquitoes through observations of haltere path and wing form.

The flapping amplitude of a flapping haltere is not small, and as such falls outside the governing assumptions of most theoretical studies of drag on a spheres in unsteady motion ${ }^{37,38}$. However, we may approximate the magnitude of form drag and virtual mass forces of a sphere in unsteady motion for comparison to the value given by Eq. (2). Boussinesq (1885) and Basset (1888) show that unsteady drag force for spheres in unsteady motion the Navier-Stokes equations give,

$$
F_{\mathrm{D}, \mathrm{u}}=6 \pi \rho \nu R V+\frac{1}{2} m_{\mathrm{g} a s} \frac{\mathrm{d} V}{\mathrm{~d} t}+6 \rho(\pi \nu)^{\frac{1}{2}} R^{2} \int_{0}^{t} \frac{f(t-\tau)}{\tau^{\frac{1}{2}}} \mathrm{~d} \tau
$$

where kinematic viscosity $\nu=\mu / \rho$, and $f(t-\tau)=\mathrm{d} V / \mathrm{d} t$, and $m_{\text {gas }}$ is the mass of gas occupying the same volume as a haltere modeled as a sphere ${ }^{37}$. The first term is form drag due to steady motion, the second is the virtual mass force arising from unsteady motion, and the third is drag from history of motion. Eq. (4) reduces to the drag equation given by Stokes (1851) for simple harmonic motion ${ }^{39}$,

$$
F_{\mathrm{D}, \mathrm{u}}=6 \pi \nu \rho R\left[1+\left(\omega R^{2} / 2 \nu\right)^{\frac{1}{2}}\right] V+\frac{1}{2} m_{\text {gas }}\left[1+\frac{9}{2}\left(2 \nu / \omega R^{2}\right)^{\frac{1}{2}}\right] \frac{\mathrm{d} V}{\mathrm{~d} t} .
$$

As before, we calculate the change peak force on the flapping halteres by increasing medium density $\rho=2.0 \mathrm{~kg} / \mathrm{m}^{3}$ from that of standard air. The anomalous peak force of a sphere in simple harmonic motion $\Delta F_{\mathrm{D}, \mathrm{u}}=17.0 \mathrm{nN}$, very close to the estimate $\Delta F_{\mathrm{D}, \mathrm{s}}$ achieved by steady drag analysis. The values of $\Delta F_{\mathrm{D}, \mathrm{s}}$ and $\Delta F_{\mathrm{D}, \mathrm{u}}$ are $0.06 \%$ and $0.08 \%$ the weight of a mosquito respectively, nearly equal to the haltere weight, estimated by volume to be $900 \mathrm{ng}$ using the density of water ${ }^{40}$.

Based on our observations of flight failure, we hypothesize the calculated anomalous aerodynamic force of $12.4-17.0 \mathrm{nN}$ in the high density medium is sufficient to incapacitate the haltere's function. In terms of haltere anatomy, such aerodynamic forces translate to increased strain detected by the campaniform sensilla embedded at the haltere shaft's base. A cantilever beam' strain $\epsilon \sim P$, where $P$ is a point force at the tip. Therefore, the increased drag force corresponds to a $67 \%$ increase in strain registered at the base. In this case, the finely tuned haltere becomes slightly out-of-sync with the wing's steering muscles, which in turn creates the rapid pitching and rolling behaviors observed in flight. 


\subsection{Flight failure due to artificially increasing haltere mass}

To test our hypothesis that aerodynamic forces of $12.4-17.0 \mathrm{nN}$ incapacitates halteres, we perform experiments with mass added to both halteres. Mass added to halteres increases the haltere inertial force $F_{i}$, which according to Eq. (1), increases the haltere's force in a similar fashion to their immersion in fog. Though increased drag and mass addition have very different dynamical effects, they both increase the strain registered by the campaniform sensilla embedded in the haltere shaft.

We begin by adding a large mass of glue $(50-150 \mathrm{ng})$ to one haltere of a mosquito $(\mathrm{N}=5)$. If weight is added to only one of the halteres, a mosquito can remain aloft. Presumably, it ignores the sensory feedback of the weighted haltere and maintains flight.

By weighting both halteres for each of 5 mosquitoes with a similar mass of glue, we observe they cannot fly. By drastically reducing the volume of glue, we determine the minimum amount added that precludes flight. A mass of glue $\sim 9.8 \pm 8.9 \mathrm{ng}(\mathrm{N}=4)$ added to a single haltere is at the cusp of complete loss of flight ability. At decreasing levels of glue $(<5 \mathrm{ng})$, a mosquito may still be able to remain aloft in our container, but must use the walls of its container as a guide for ascent.

With sufficient glue added, mosquitoes pitch and roll as they would in a denser-than-air gas or if both halteres had been ablated. The third column in Fig.2 depicts a mosquito flying in normal air with glue on its halteres. The glue is shaded in blue in Fig.4. As seen in Fig.2c, a mosquito with glued halteres also falls quickly.

The anomalous inertial force added to the halteres by glue is $\Delta F_{\mathrm{i}}=m_{\text {glue }} A \ell(2 \pi f)^{2}$. The mass of the glue $m_{\text {glue }}$ is calculated based on volume estimated from images. For glue of mass $m_{\text {glue }}=9.8 \mathrm{ng}$, the maximum additional inertial force is $\Delta F_{\mathrm{i}}=13.6 \mathrm{nN}$, which is similar to the haltere's aerodynamic force in fog, calculated in $\S 2.2$. The similarity of these two values suggest that indeed the aerodynamic force of fog on halteres is responsible for flight failure.

While the addition of mass to the halteres will do little to change $\Delta F_{\mathrm{s}}$ and $\Delta F_{\mathrm{u}}$, it will alter the Coriolis force measured by a rotating mosquito, thereby affecting $F_{\text {haltere }}$. The change in magnitude of the Coriolis force via glue addition may be expressed as

$$
\Delta F_{\mathrm{C}}=2 m_{\text {glue }} \Omega V_{\max }
$$

where $\Omega$ is the angular velocity of the mosquito's body. However, this force is small compared with $\Delta F_{\mathrm{i}}$. Consider the mosquito in Fig.1d, rotating at $48 \mathrm{rad} / \mathrm{s}$, an unusually high rotation rate in our observations. Using a mass of glue of $m_{\text {glue }}=19 \mathrm{ng}$, yields a change in Coriolis force $\Delta F_{\mathrm{C}}=1.35$ $\mathrm{nN}$, which is an order of magnitude lower than the change in inertial force. Although halteres are very sensitive, it is unlikely changes in Coriolis forces equivalent to $0.007 \%$ the weight of a mosquito have a substantial effect on flight stability.

Both the introduction of heavy gas and the treatment using glue each provide the halteres an additional resistance force. We thus pinpoint the cause of flight failure of a mosquito in fog to malfunctioning of the haltere system, and not the wings. We speculate this anomalous force places an additional strain on the halteres during flapping. Consequently, the mosquitoes lose the ability to successfully engage steering muscles, causing them to unintentionally turn upside down. A more detailed mechanism involving the pathways from halteres to muscles in unclear. While we do not have a definitive mechanism for flight failure in hyperdense mediums, we can pinpoint the failure as a collapse in haltere function. Next, we show how the additional mass of glue can change the free vibration parameters of the halteres. 


\subsection{Haltere mass affects their natural frequency}

All vibratory systems, physical or biological, have a natural frequency due to the geometry and elasticity of their components. Halteres are likely flapped at, or close to, their natural frequency or one of their resonance modes, to reduce the power required to reach maximum amplitude. Wings and halteres are meant to flap together, but if the vibration properties change due to haltere mass addition, a subtle mismatch in phase may occur between wings and halteres. In this section, we examine how increased mass to the haltere affects its natural frequency.

We pluck the halteres of 10 freshly dead mosquitoes, and allow them to vibrate freely (Fig.5a, Supp. Video 4). As seen in Fig.5b, the heavier amounts of glue (40-50 ng) decrease the damped natural frequency $\omega_{\mathrm{d}}$ by $14 \%$ as one might expect when increasing a cantilever's inertia. Small amounts of glue $(5-15 \mathrm{ng})$ increase $\omega_{\mathrm{d}}$ by $15 \%$ relative to an unaltered haltere. The cause for this increase in frequency is unknown. We find the above differences for $\omega_{\mathrm{d}}$ to be statistically significant, rendering $\mathrm{P}$-values of 0.02 when compared to the unaltered haltere sample.

As shown by the examples in Fig.5c,d, all of the freely vibrating halteres tested have asymmetrical exponential decay envelopes, likely the result of a single muscle which drives haltere oscillation ${ }^{32}$ alongside a thoracic oscillator ${ }^{41}$ when the mosquito is flying. The lower bound of the envelope in Fig.5c, (dotted), levels off more quickly than the upper bound (solid), indicating preferential deflection towards the mosquito's anterior surface. The addition of glue to the haltere (Fig.5d), not only decreases the resonance frequency of the haltere, from $252 \mathrm{~Hz}$ in Fig.5c to $205 \mathrm{~Hz}$ in Fig.5d, but slows the rate by which the vibrations decay. The changes in vibration frequencies and envelopes in plucked halteres resulting from glue addition, suggests a subtle disruption to haltere kinematics in flight. Altered kinematics and mass distribution will impact strain measurements, made by the haltere's campaniform sensilla, and beat rhythm, rendering halteres less effective in regulating wing motion.

\section{Discussion}

Our study highlights the sensitivity and precision of mosquito halteres. We induce flight failure in mosquitoes using two independent means, immersion in hyperdense flight environments and addition of $0.2 \%$ mass to the halteres. Our result shows haltere systems are highly tuned to the properties of normal air, rendering small changes to these aerodynamic properties devastating to the insect. A greater understanding of the sensitivity of such sensing systems may lead to a greater understanding of the complex control system of insects and new ways to reduce their populations.

In tests with other insects, we found haltere size is inversely related to their vulnerability in fog. Fruitflies Drosophila melanogaster and Drosophila virilis have halteres smaller than that of a mosquito. According to our observations, they also fail to fly in dense fog. Conversely, houseflies (Musca domestica) with larger halteres, can stay aloft in dense fog (Supp. Video 5), presumably because their flight system does not require such a finely tuned central pattern generator.

Diptera are not alone in possessing very specialized and tuned sensing organs. The male Emperor moth has chemoreceptors which can smell a female $11 \mathrm{~km}$ away ${ }^{42}$. A squid's head can detect vibration, and in the $75-100 \mathrm{~Hz}$ range, it can sense $0.2 \mu \mathrm{m}$ peak-to-peak water displacement ${ }^{43}$. Some snakes are able to sense vibrations of their head as small as $0.1 \mathrm{~nm}$ peak-to-peak amplitude. Homing pigeons can detect sound frequencies as low as $0.05 \mathrm{~Hz}$, while bats can detect frequencies as high as $100 \mathrm{kHz}^{43}$. In light of the extreme sensitivity in sensing ability in the animal kingdom, disruption of mosquito haltere function with only a $2 \mathrm{ng}$ addition of glue appears more feasible.

When compared to human anatomy, halteres most closely resemble the vestibular structure in our inner ear, used for balance. The inner ear is susceptible to infections, altering the inner 
ear's original state slightly, causing balance disorders ${ }^{44}$. The vestibular fluid in the semicircular canals of the inner ear pushes against a small membrane, the cupula, as we move our heads. It has been calculated that cupulas have a maximum displacement of 3 microns, or $0.4 \%$ the amplitude displacement of a mosquito haltere ${ }^{45}$. Indeed, microscopic precision in sensing, and the accompanying vulnerability, is a commonality among organisms.

Mosquitoes are not alone in being affected by extreme flight medium conditions. Altering flight medium characteristics reduces the flight performance of most flying creatures. Birds at high altitudes fly with reduced forward drag, but increased requirements for lift production ${ }^{46}$. Orchid bees hovering in heliox $\left(80 \% \mathrm{He} / 20 \% \mathrm{O}_{2}\right)$, with a density of $0.40 \mathrm{~kg} / \mathrm{m}^{3}$, experienced $50 \%$ increases in lift coefficients relative to normal air ${ }^{47}$. The decrease of flight ability in low density and hypoxic environments is a result of both aerodynamics and metabolism. The dynamic viscosity of the flight medium might also create instability flight. It is not possible to calculate the viscosity of our mixtures of R134a and air without further experiments ${ }^{48}$, though based on the viscosities of R134a (12 $\mu \mathrm{Pa}$-s) and air (19 $\mu \mathrm{Pa}-\mathrm{s})$, we do not expect the viscosity of the mixture to be dramatically different than that of air. For example, a mixture of nitrogen and R-12 (Freon) with the same volume fraction we use to a create a flight medium density $\rho=2 \mathrm{~kg} / \mathrm{m}^{3}$ (74\% air, $\left.26 \% \mathrm{R} 134 \mathrm{a}\right)$ has a viscosity of $16 \mu \mathrm{Pa}-\mathrm{s}^{48}$.

While halteres are extremely sensitive, a Reynolds number of order ten indicates inertial effects dominate viscous effects, so changes in viscosity should have minimal effect. In the case of glue addition, the added bulk induces instability via inertial forces. Efforts were made to deposit the glue at the extreme tips of halteres, but the variation in glue mass to induce failure, $\sim 9.8 \pm 8.9 \mathrm{ng}$, may be caused by deposition location. Haltere size variation may also play a role, and we would expect smaller halteres to be more sensitive to mass addition.

The introduction of gases other than air, displaces oxygen needed for breathing and raises the possibility of hypoxia influencing the results in our experiments. At a density of $2 \mathrm{~kg} / \mathrm{m}^{3}$, the threshold at which no mosquito can fly, the oxygen in our container dropped from $21 \%$ by volume to $15 \%$ with the introduction of R134a. Such conditions are not prohibitive to flight: insects have been known to survive extreme hypoxic conditions ${ }^{49}$ and colonize high altitudes above $6000 \mathrm{~m}$ where the oxygen content is a mere $9.5 \%$. In our observations, mosquitoes left in containers where R134a was used to produce densities greater than $\sim 3 \mathrm{~kg} / \mathrm{m}^{3}$ would lapse into unconsciousness within $10 \mathrm{~s}$, but no sooner than $4 \mathrm{~s}$. In our observations, insects will cease flapping immediately upon unconsciousness. Since the falling mosquitoes in our experiments continued flapping during descent, we are confident hypoxia did not play a role in our results. Even at the highest gas densities, flight failure behavior occurs within a time much less than $1 \mathrm{~s}$ following a mosquito's drop into a container, whereas the total flight time is less than $2 \mathrm{~s}$. The fast onset of flight failure and the flapping of the mosquitoes suggest they are indeed conscious and attempting to fly in our experiments.

Due to the water repellent nature of a mosquito's body, including the halteres, fog particles are unlikely to deposit and remain attached to flapping wings, halteres, and other sensors in quantities sufficient to hinder flight. As seen in Fig.4 the rapidly moving appendages have tightly-spaced feather-like sensilla. However, a wetting liquid, such as nontoxic soybean oil (density of $920 \mathrm{~kg} / \mathrm{m}^{3}$ ), a common insecticide base, may adhere to halteres. Oil-based insecticides are known to be produced in a range of $\operatorname{sizes}^{50}, 2-110 \mu \mathrm{m}$ in diameter. A single oil droplet $10 \mu \mathrm{m}$ in diameter deposited on each haltere would be sufficient to ground a mosquito. Production of insecticide-free fogs may be a viable mosquito-control strategy in some situations. We note that with current foggers, mosquitoes and similarly sized insects will be downed near the output nozzle, not due to immediate insecticide absorption, but due to the fog cloud's density.

To eliminate the likelihood of fog interrupting the function of the mosquitoes' antennae, we 
cropped the antennae at the base and observed they were able to maintain qualitatively normal flight in a container. Fog exposure on mosquitoes without antennae was met with grounding, in a manner similar to those with intact organs.

Mosquito control using fog produced by ultrasonic humidifiers is not currently viable, especially for large areas. Water and electricity consumption will be unreasonable in most environments, when compared to traditional insecticide sprays. The most realistic use of water droplets to protect humans against mosquitoes would consist of a continuously falling curtain of microscopic droplets over thresholds such as windows and doors. Similar "air doors" are currently used, such that a sheet of high velocity air is directed downward from the top of a doorway. While such curtains of air are effective as an insect filter, they require high power and are noisy. In places where water is plentiful, a "fog door" would provide protection with no sound and lower operating costs.

\section{Methods}

\section{Mosquito Source and Care}

Male and female Anopheles freeborni mosquitoes are used in this study, and are reared and provided by the Centers for Disease Control (CDC) in Atlanta, GA. Mosquitoes are adults upon delivery and are fed a nectar solution prepared by the CDC. We observe no difference in flight failure characteristics between male and female mosquitoes, and no attempt was made to separate the sexes following initial experiments.

\section{Mosquito Handling}

Mosquitoes are transferred to various containers with a John Hock brand aspirator. They are singularly held in place by one of two methods. The first method is by a continuous vacuum pen (Virtual Industries Tweezer Vac) which can pick up and release mosquitoes without removing appendages or rupturing their exoskeleton. The second method, used in tethered experiments, employs liquified wax to attach them to a tether, which is preferable for long-term restraint, as it places no external forces on the exoskeleton. Tethering with wax just behind the head or performing other tedious procedures, such as glue deposition on the halteres, requires the mosquitoes to be incapacitated by inhalation of $\mathrm{CO}_{2}$. Short exposure to the gas anesthetizes insects, while prolonged exposure causes death. Tethering requires the mosquitoes be exposed to $\mathrm{CO}_{2}$ for 10 seconds, to remain inactive for roughly 20 seconds.

\section{Filming}

A Phantom V210 high-speed camera is the primary tool for observing of mosquito flight, and we

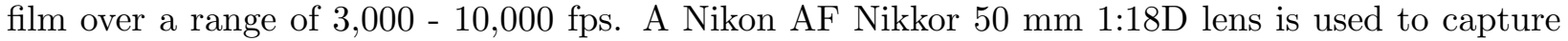
the entire flight arena, while a Navitar 1-60135 is used for macro filming. Flight arenas are lit by 4, low temperature LED's (IDT Honeycomb LED-1). Measurement and tracking within videos is done with Tracker, an open source physics program. All reported P-values in this manuscript are results of an unpaired t-test application to our data.

\section{Fog Experiments}

Fog is produced with an Air O Swiss 7145 consumer humidifier with continuous adjustability in fog density. A hose attached to the humidifier directs the stream of mist to the subject. A droplet sizing instrument (DC-III; KLD Labs Inc., New York, NY) is used to characterize the spectra of droplets generated by our humidifiers. As seen in Fig.6, a miniature wind tunnel $(7.5 \mathrm{~cm} \mathrm{x} 5 \mathrm{~cm}$ $\mathrm{x} 11 \mathrm{~cm}$ ) has been constructed to deliver fog to a mosquito's contained, flight environment. It uses 
a variable speed computer fan to pull air through a $6.5 \mathrm{~cm} \mathrm{x} 1 \mathrm{~cm}$ opening, which uses a square grate to laminarize the incoming air and contain mosquitoes. The air speed at the inlet is $3 \mathrm{~cm} / \mathrm{s}$, measured by particle tracking. Control experiments indicate no substantial effect of incoming air on mosquito flight. Flight is evoked from the mosquitoes within by human breath into the tunnel's intake. Human breath excites both male and female Anopheles freeborni.

\section{Dense Gas Experiments}

For experiments with flight in various gas densities, R134a (1,1,1,2-Tetrafluoroethane), a non-toxic gas $^{51}$, with a density of $4.25 \mathrm{~kg} / \mathrm{m}^{3}$ at standard temperature and pressure, is piped into a sealed, transparent, semi-circular container of height $15 \mathrm{~cm}$ and width $5.5 \mathrm{~cm}$. Mosquitoes were capable of indefinite perching on the interior walls and ends of the container post-experiment. Subjects used in R134a-based experiments are still alive the day following exposure to R134a, when sufficient ambient oxygen is allowed into their container. The density of the gas mixture inside the container is determined by the container's mass reading on an analytical balance. Atmospheric conditions inside our lab remain with $5{ }^{\circ} \mathrm{C}$ range. Mosquitoes were introduced through a hole in the top of the container, and allowed to fly freely, or fall to the bottom.

\section{Haltere Augmentation Experiments}

To investigate the effect of altered forcing on mosquito halteres, we laden the halteres of untethered, anesthetized mosquitoes with UV curable glue with density $\rho_{\text {glue }}=960 \mathrm{~kg} / \mathrm{m}^{3}$, a the haltere tip. The glue's density is measured by massing a disc of cured glue whose volume is measured by digital calipers. The glue is deposited onto unconscious mosquitoes via a thin wire, by hand, under a microscope. Glue is cured with a UV flashlight. Mosquitoes are given 10 minutes to recover from anesthesia and released for observation. Mosquitoes anesthetized by the same manner as those with glue on halteres recovered to resume qualitatively normal flight after approximately 5 minutes in atmospheric air. Post-flight, mosquitoes are imaged in a Phenom Pro G2 scanning electron microscope (SEM), which provides a reference scale in frame, to measure the volume of glue deposited. Glue dollops are traced with Tracker to calculate the irregular shape's area and thickness.

\section{Conclusion}

We perform flight experiments with mosquitoes in mediums of varying fluid density. In gases with densities twice that of standard air, mosquitoes suffer rapid pitching, rolling, and ultimately grounding. Though no directly applicable fluid dynamic model exists, we estimate drag forces on the halteres flapping in dense air using steady and unsteady drag models, which provide comparable values. We hypothesize the anomalous aerodynamic drag forces, on the order of $10 \mathrm{nN}$, disrupt the rhythm of mosquito halteres, their gyroscopic sensors and wingbeat pattern generators. We test this hypothesis by adding weight to the halteres to create inertial forces on the same order of magnitude as aerodynamic forces in heavy air. An increase in the haltere's mass by $10 \mathrm{ng}$, is sufficient to disable flight. We also show weighted halteres have different natural frequencies than unaltered halteres, and suggest this change in natural frequency prevents synchronization between wings and halteres. This study shows halteres are highly tuned to the properties of air, making changes in air density a potential method to be applied in mosquito control. 


\section{Acknowledgements}

We thank Caitlin Moyer, Lee-Ellen Thornton, and Luke Telljohann for their early experimental contributions, Michael Leamy and Alper Erturk for suggestions regarding haltere vibration, Muhammad Farooq for droplet size data, and NSF (PHY-0848894) for financial support.

\section{References}

[1] World malaria report : 2011. Tech. Rep., World Health Organization (2011).

[2] Becker, N. et al. Mosquitoes and their control, vol. 2 (Springer, 2010).

[3] Focks, D. A., Brenner, R. J., Hayes, J. \& Daniels, E. Transmission thresholds for dengue in terms of aedes aegypti pupae per person with discussion of their utility in source reduction efforts. The American Journal of Tropical Medicine and Hygiene 62, 11-18 (2000).

[4] Lengeler, C. Insecticide-treated bed nets and curtains for preventing malaria. Cochrane Database System 2 (2004).

[5] Pates, H. \& Curtis, C. Mosquito behavior and vector control. Annual Review of Entomology 50, 53-70 (2005).

[6] Borovsky, D. Trypsin-modulating oostatic factor: a potential new larvicide for mosquito control. Journal of Experimental Biology 206, 3869-3875 (2003).

[7] Britch, S. C. et al. Evaluation of ulv and thermal fog mosquito control applications in temperate and desert environments. Journal of the American Mosquito Control Association 26, 183-197 (2010).

[8] Khasnis, A. \& Nettleman, M. Global warming and infectious disease. Archives of Medical Research 36, 689-696 (2005).

[9] Voigt, C., Schneeberger, K., Voigt-Heucke, S. \& Lewanzik, D. Rain increases the energy cost of bat flight. Biology letters 7, 793-795 (2011).

[10] Dickerson, A., Shankles, P., Madhavan, N. \& Hu, D. Mosquitoes survive raindrop collisions by virtue of their low mass. Proceedings of the National Academy of Sciences 109, 9822-9827 (2012).

[11] Pinnick, R. et al. Vertical structure in atmospheric fog and haze and its effects on visible and infrared extinction. Journal of the Atmospheric Sciences 35, 2020-2032 (1978).

[12] Gultepe, I. et al. The fog remote sensing and modeling field project. Bulletin of the American Meteorological Society 90, 341-359 (2009).

[13] Sinclair, D. \& La Mer, V. Light scattering as a measure of particle size in aerosols. the production of monodisperse aerosols. Chemical Reviews 44, 245-267 (1949).

[14] Thompson, B., Ward, J. \& Zinky, W. Application of hologram techniques for particle size analysis. Applied optics 6, 519-526 (1967). 
[15] Seiber, J., Wilson, B. \& McChesney, M. Air and fog deposition residues of four organophosphate insecticides used on dormant orchards in the san joaquin valley, california. Environmental Science \& Technology 27, 2236-2243 (1993).

[16] Bevanger, K. Bird interactions with utility structures: collision and electrocution, causes and mitigating measures. Ibis 136, 412-425 (1994).

[17] Bender, J. \& Dickinson, M. A comparison of visual and haltere-mediated feedback in the control of body saccades in drosophila melanogaster. Journal of Experimental Biology 209, 4597-4606 (2006).

[18] Götz, K. Flight control in drosophila by visual perception of motion. Biological Cybernetics 4, 199-208 (1968).

[19] Cheng, B., Deng, X. \& Hedrick, T. The mechanics and control of pitching manoeuvres in a freely flying hawkmoth (manduca sexta). The Journal of Experimental Biology 214, 4092-4106 (2011).

[20] Arbas, E. Control of hindlimb posture by wind-sensitive hairs and antennae during locust flight. Journal of Comparative Physiology A: Neuroethology, Sensory, Neural, and Behavioral Physiology 159, 849-857 (1986).

[21] Niehaus, M. Flight and flight control by the antennae in the small tortoiseshell (aglais urticae l., lepidoptera). Journal of Comparative Physiology A: Neuroethology, Sensory, Neural, and Behavioral Physiology 145, 257-264 (1981).

[22] Gewecke, M. \& Niehaus, M. Flight and flight control by the antennae in the small tortoiseshell (aglais urticae l., lepidoptera). Journal of Comparative Physiology A: Neuroethology, Sensory, Neural, and Behavioral Physiology 145, 249-256 (1981).

[23] Sane, S., Dieudonné, A., Willis, M. \& Daniel, T. Antennal mechanosensors mediate flight control in moths. Science Signalling 315, 863 (2007).

[24] Böröczky, K., Wada-Katsumata, A., Batchelor, D., Zhukovskaya, M. \& Schal, C. Insects groom their antennae to enhance olfactory acuity. Proceedings of the National Academy of Sciences 110, 3615-3620 (2013).

[25] Sutcliffe, J. F. Sensory bases of attractancy: morphology of mosquito olfactory sensilla-a review. Journal of the American Mosquito Control Association 10, 309 (1994).

[26] Fraenkel, G. \& Pringle, J. Biological sciences: Halteres of flies as gyroscopic organs of equilibrium. Nature 141, 919-920 (1938).

[27] Wu, W., Wood, R. \& Fearing, R. Halteres for the micromechanical flying insect. In Robotics and Automation, 2002. Proceedings. ICRA'02. IEEE International Conference on, vol. 1, 6065 (IEEE, 2002).

[28] Dickinson, M. Come fly with me. Engineering and Science 66, 10-19 (2003).

[29] Bartussek, J., Mutlu, A. K., Zapotocky, M. \& Fry, S. N. Limit-cycle-based control of the myogenic wingbeat rhythm in the fruit fly drosophila. Journal of The Royal Society Interface 10 (2013). 
[30] Sane, S. P. \& McHenry, M. J. The biomechanics of sensory organs. Integrative and Comparative Biology 49, i8-i23 (2009).

[31] Sellke, K. et al. Biological and morphological studies on harmful tipulids. Zeitschrift fur wissenschaftliche Zoologie 148, 465-555 (1936).

[32] Pringle, J. The gyroscopic mechanism of the halteres of diptera. Philosophical Transactions of the Royal Society of London. Series B, Biological Sciences 347-384 (1948).

[33] Heide, G. Neural mechanisms of flight control in diptera. Biona-report 2, 35-52 (1983).

[34] Fayyazuddin, A. \& Dickinson, M. Convergent mechanosensory input structures the firing phase of a steering motor neuron in the blowfly, calliphora. Journal of Neurophysiology 82, 1916-1926 (1999).

[35] Paaijmans, K. P. \& Thomas, M. B. The influence of mosquito resting behaviour and associated microclimate for malaria risk. Malaria Journal 10, 183 (2011).

[36] Pantaleone, J. \& Messer, J. The added mass of a spherical projectile. American Journal of Physics 79, 1202 (2011).

[37] Karanfilian, S. \& Kotas, T. Drag on a sphere in unsteady motion in a liquid at rest. Journal of Fluid Mechanics 87, 85-96 (1978).

[38] Mei, R. Flow due to an oscillating sphere and an expression for unsteady drag on the sphere at finite reynolds number. Journal of Fluid Mechanics 270, 133-174 (1994).

[39] Stokes, G. G. On the effect of the internal friction of fluids on the motion of pendulums, vol. 9 (Pitt Press, 1851).

[40] Nalbach, G. \& Hengstenberg, R. The halteres of the blowfly calliphora. Journal of Comparative Physiology A: Neuroethology, Sensory, Neural, and Behavioral Physiology 175, 695-708 (1994).

[41] Nachtigall, W. \& Wilson, D. M. Neuro-muscular control of dipteran flight. Journal of Experimental Biology 47, 77-97 (1967).

[42] Taylor, B., Green, J. \& Farndon, J. The Big Bug Book: Discover the Amazing World of Beetles, Bugs, Butterflies, Moths, Insects and Spiders (Lorenz Books, 2004).

[43] Schmidt-Nielsen, K. Animal Physiology: Adaptation and Environment (Cambridge University Pr, 1997), 5 edn.

[44] Balance disorders (2010). URL https://www.nidcd.nih.gov/health/balance/pages/ balance_disorders.aspx.

[45] Oman, C. \& Young, L. The physiological range of pressure difference and cupula deflections in the human semicircular canal: theoretical considerations. Acta Oto-laryngologica 74, 324-331 (1972).

[46] Altshuler, D. \& Dudley, R. The physiology and biomechanics of avian flight at high altitude. Integrative and Comparative Biology 46, 62-71 (2006).

[47] Dudley, R. Extraordinary flight performance of orchid bees (apidae: Euglossini) hovering in heliox ( $80 \%$ he/20\% o2). Journal of experimental biology 198, 1065-1070 (1995). 
[48] Buddenberg, J. \& Wilke, C. Calculation of gas mixture viscosities. Industrial \& Engineering Chemistry 41, 1345-1347 (1949).

[49] Hoback, W. W. \& Stanley, D. W. Insects in hypoxia. Journal of Insect Physiology 47, 533-542 (2001).

[50] Hoffmann, W. C. et al. Spray characterization of ultra-low-volume sprayers typically used in vector control 1. Journal of the American Mosquito Control Association 25, 332-337 (2009).

[51] Alexander, D. \& Libretto, S. An overview of the toxicology of hfa-134a (1, 1, 1, 2tetrafluoroethane). Human\& Experimental Toxicology 14, 715 (1995). 

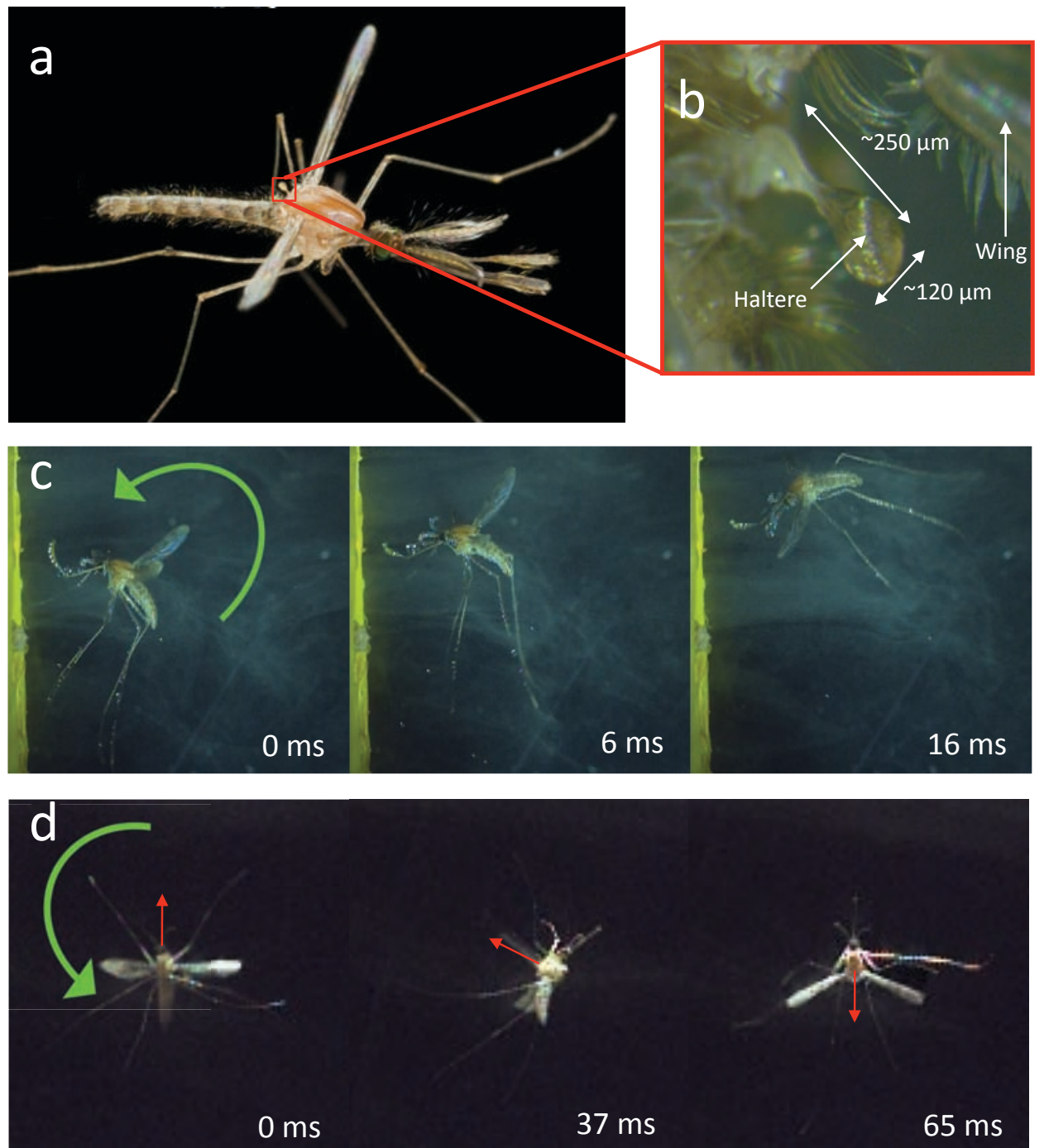

Figure 1: (a) An Anopheles freeborni mosquito in flight. The location of mosquito's haltere boxed in red. (b) A mosquito haltere. A video sequence of a mosquito pitching forward (c) and rolling (d) while aloft in a stream of fog particles. The red arrows denote the direction normal to the mosquito's back. 


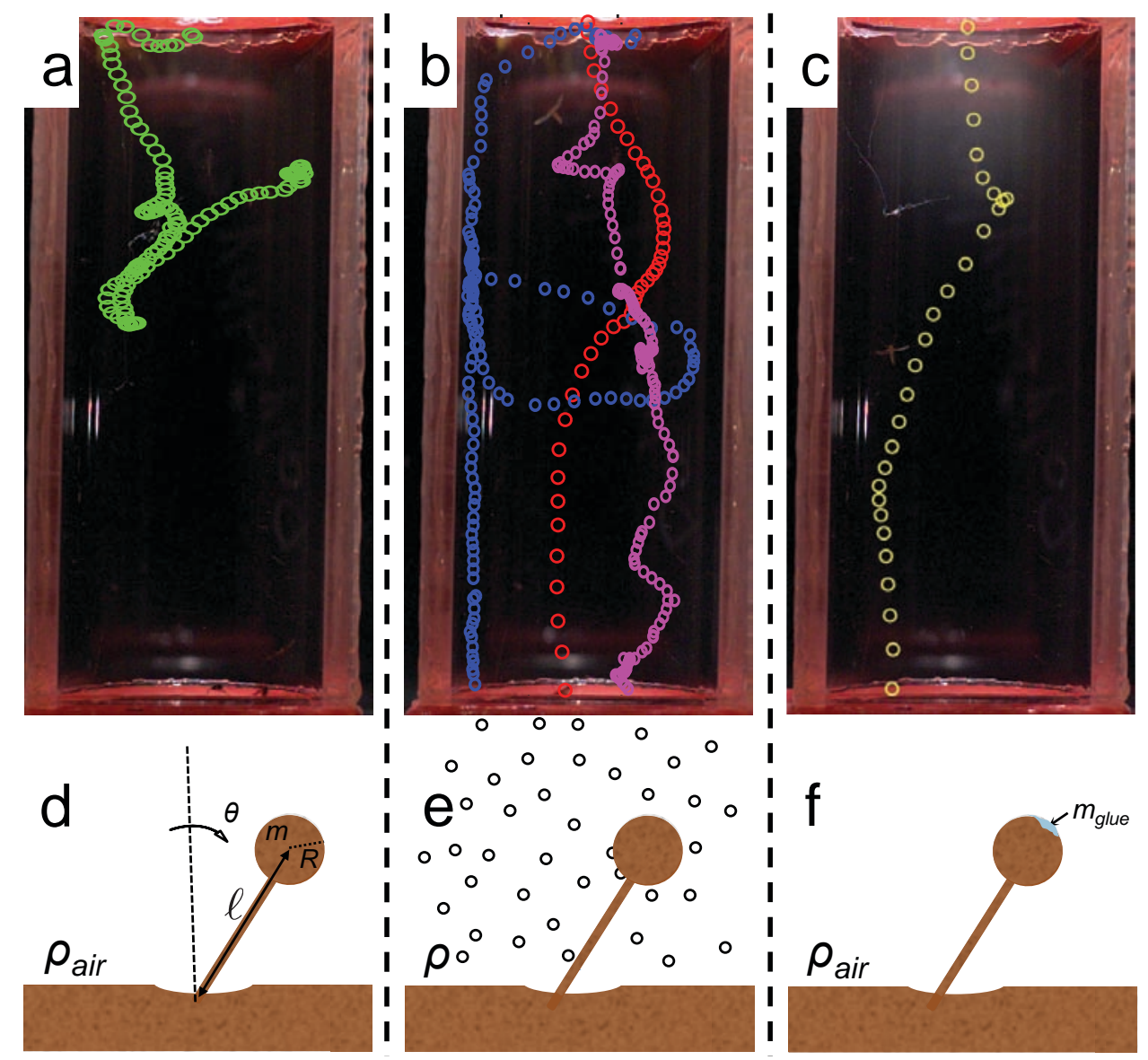

Figure 2: Three columns designate flight in normal air, fog or dense gas, and flight with glue on halteres respectively. Flight tracks (a-c) for a mosquito flying in normal air (a); in gas of density $\rho=1.75$ (blue), 1.9 (pink), 2 (red) kg/m³ (b); with glue deposited on its halteres (c). Schematics (d-f)of haltere flapping in normal air (d), in fog or heavy gas (e), with glue deposited on the tip (f). 


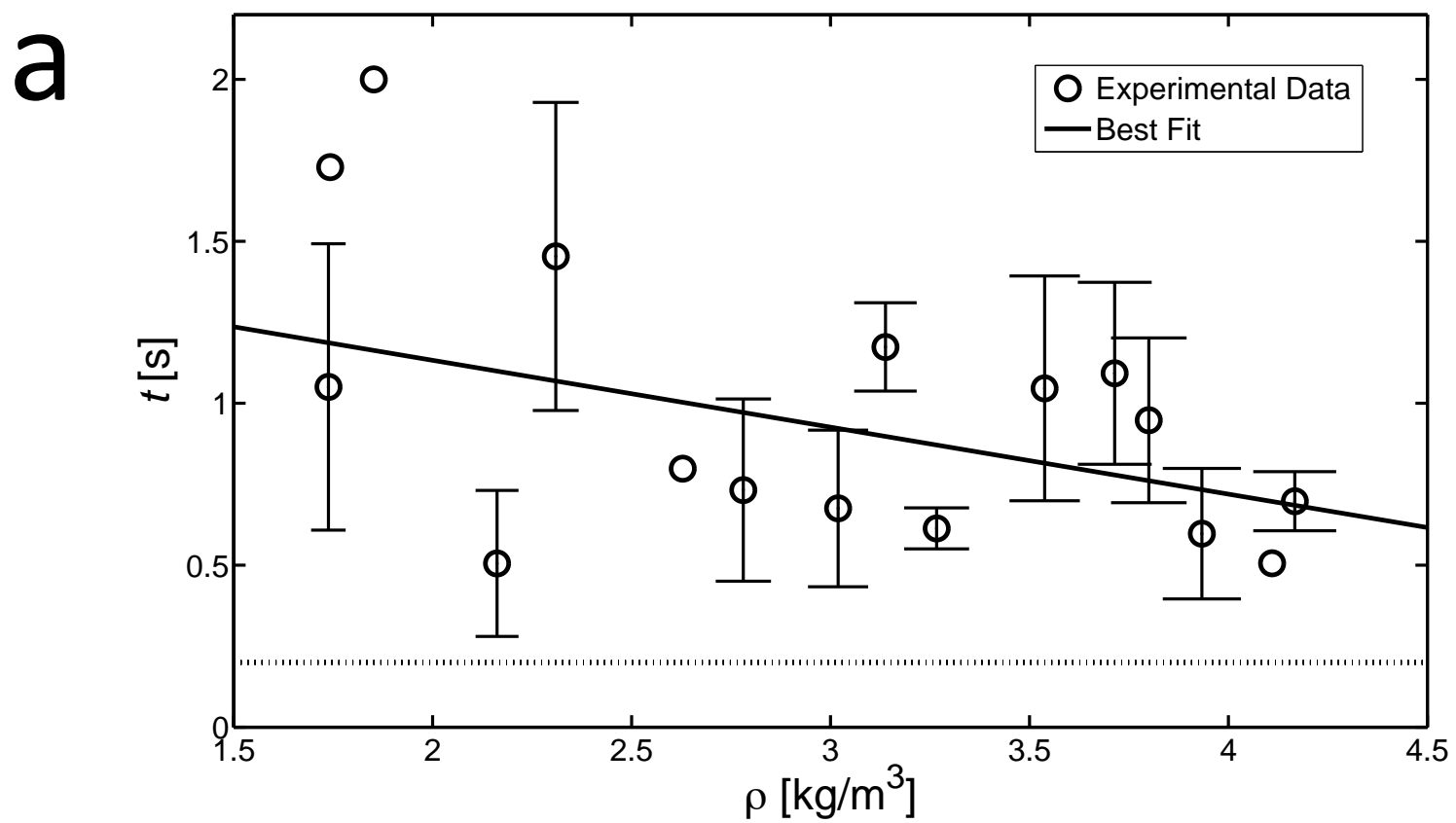

b

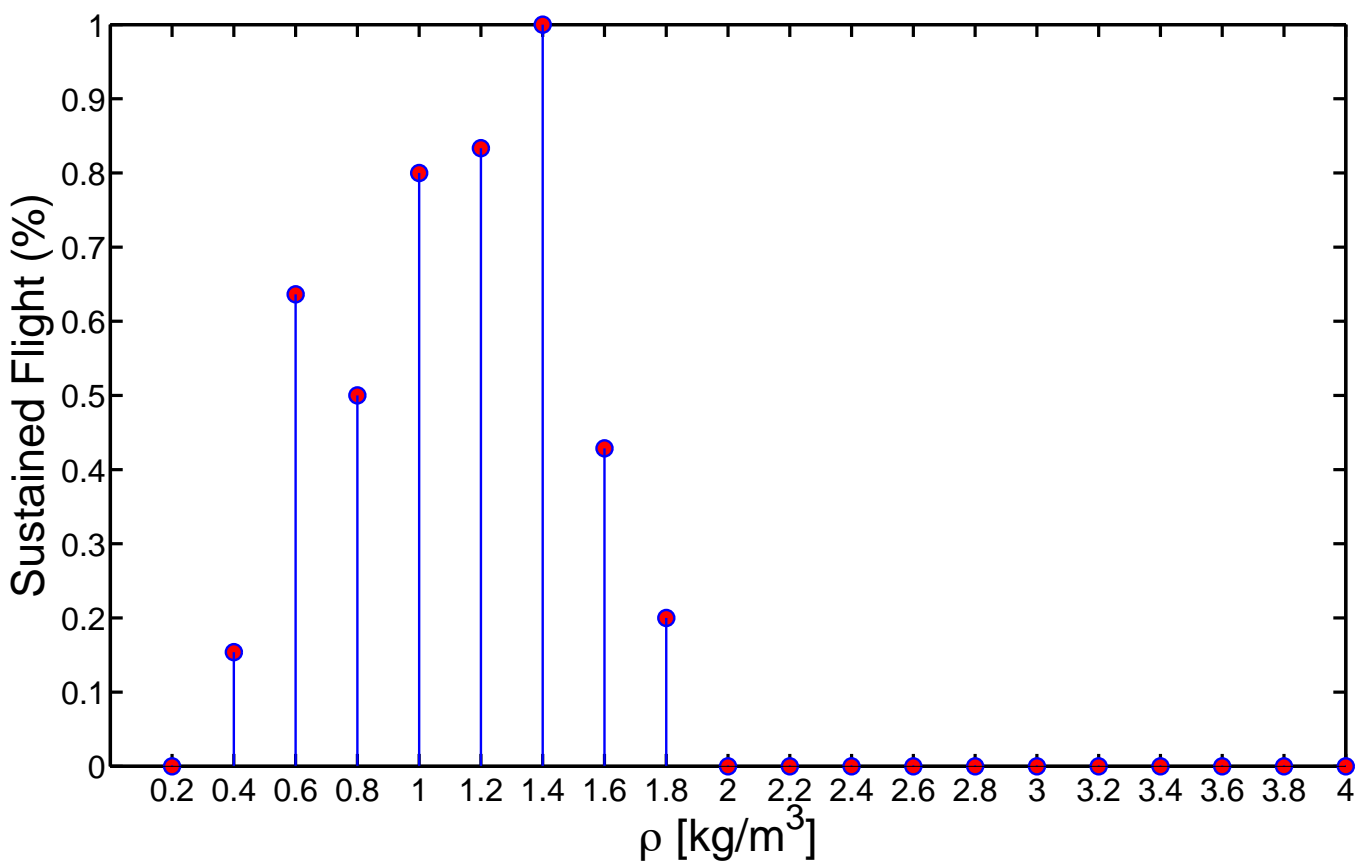

Figure 3: (a) Relation between gas density $\rho$ and descent time for mosquitoes suddenly introduced into a container of dense gas. Error bars denote \pm one standard error. Trials were performed once per animal. Points without error bars denote a single trial at the specified gas density. The dotted line a the bottom bounds the minimum time required to fall the container depth at terminal velocity. (b) Relation between gas density $\rho$ and percentage of mosquitoes able to sustain flight. Each bar represents $\mathrm{N}=4-12$ trials. 


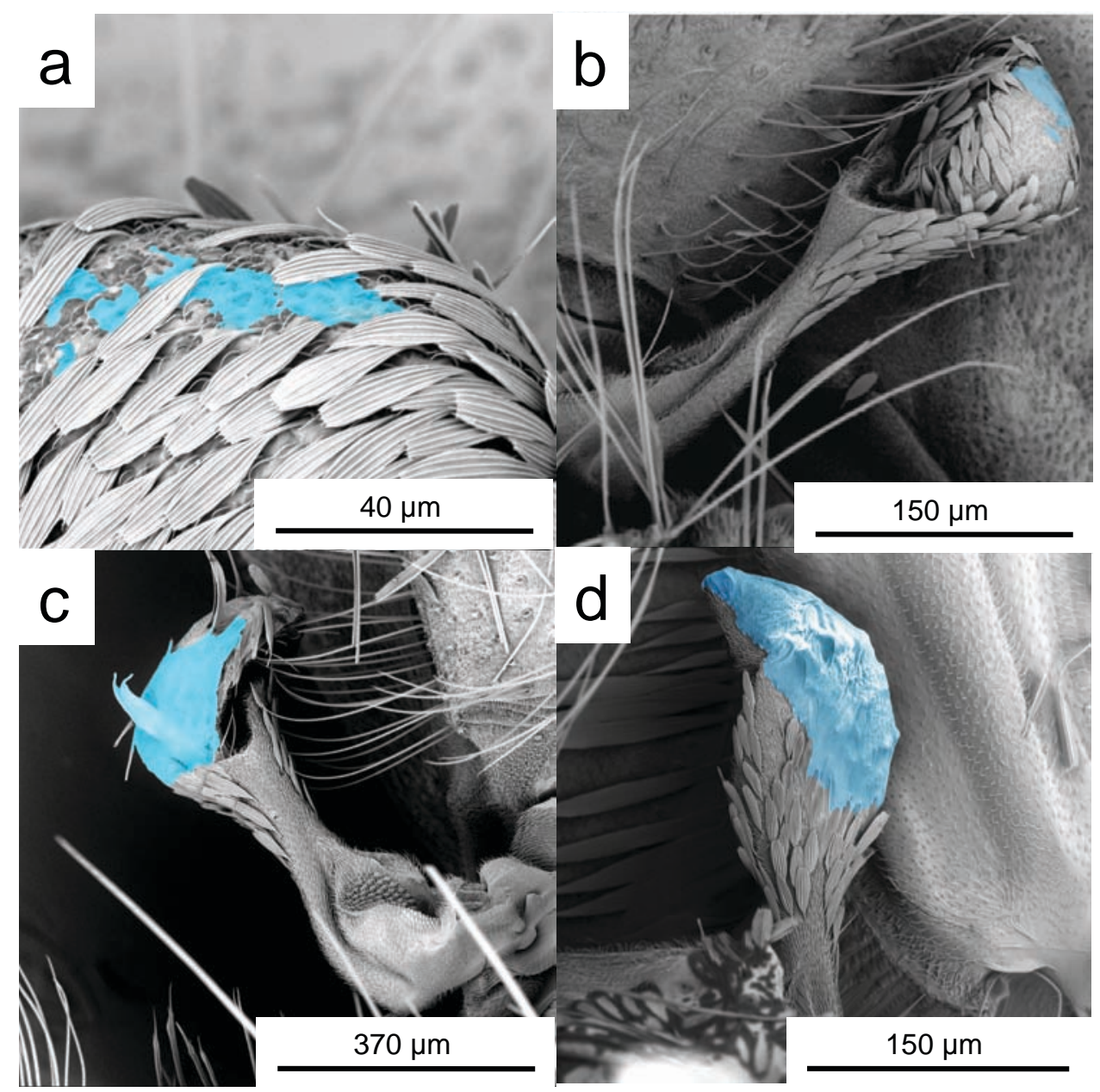

Figure 4: SEM photographs of halteres with various amount of glue, shaded in blue. The glue has a mass (a) < $1 \mathrm{ng}$, (b) $2 \mathrm{ng}$, (c) $13 \mathrm{ng}$, and (d) $20 \mathrm{ng}$. 

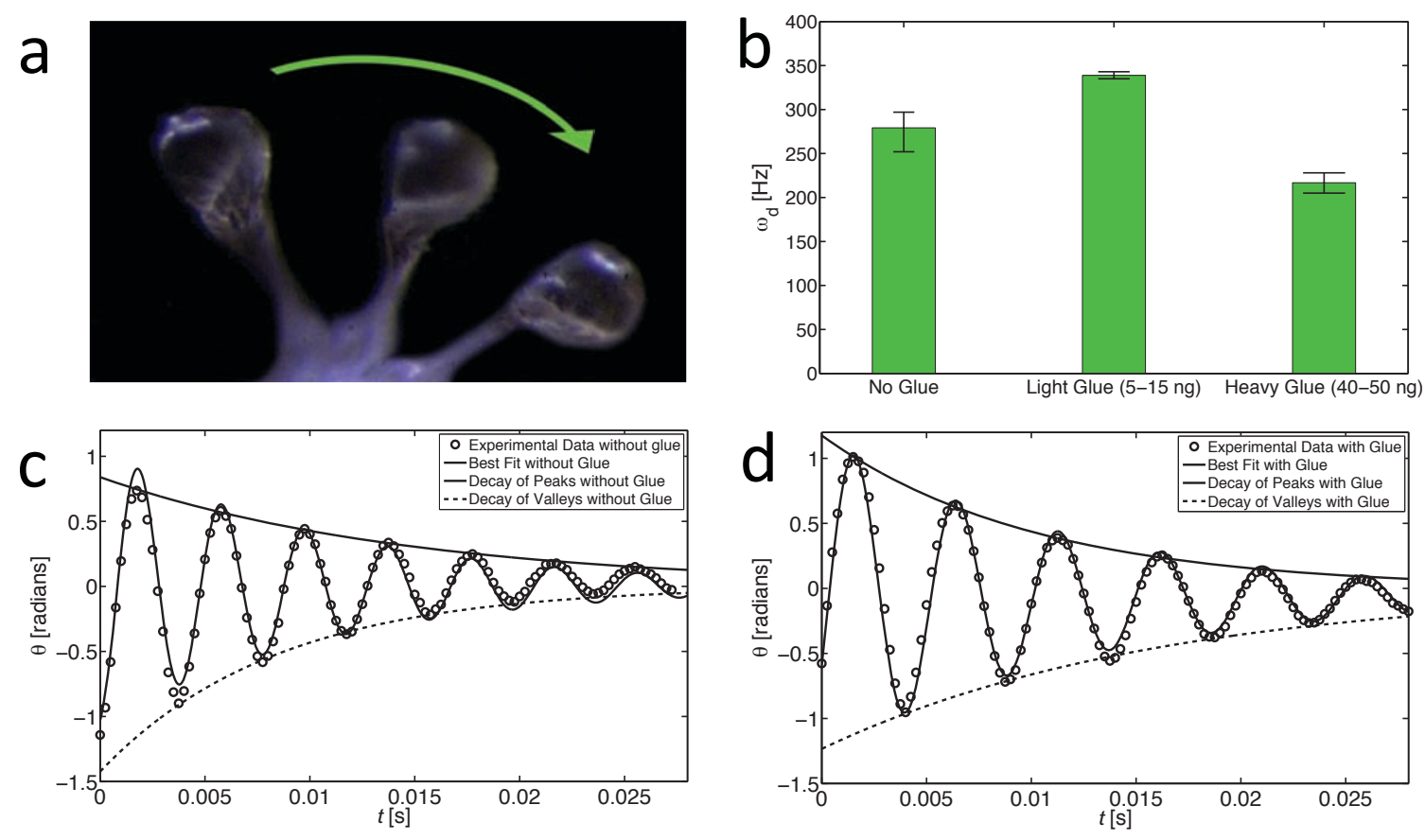

Figure 5: (a) Haltere swinging through its plane of motion after manual plucking. (b) Damped natural frequencies of freely vibrating halteres with various amount of glue added $(\mathrm{N}=8)$. The bars on each column bound the minimum and maximum values for frequency. (c) Time course of freely vibrating haltere, and (d) with approximately $50 \mathrm{ng}$ of glue deposited on the tip.

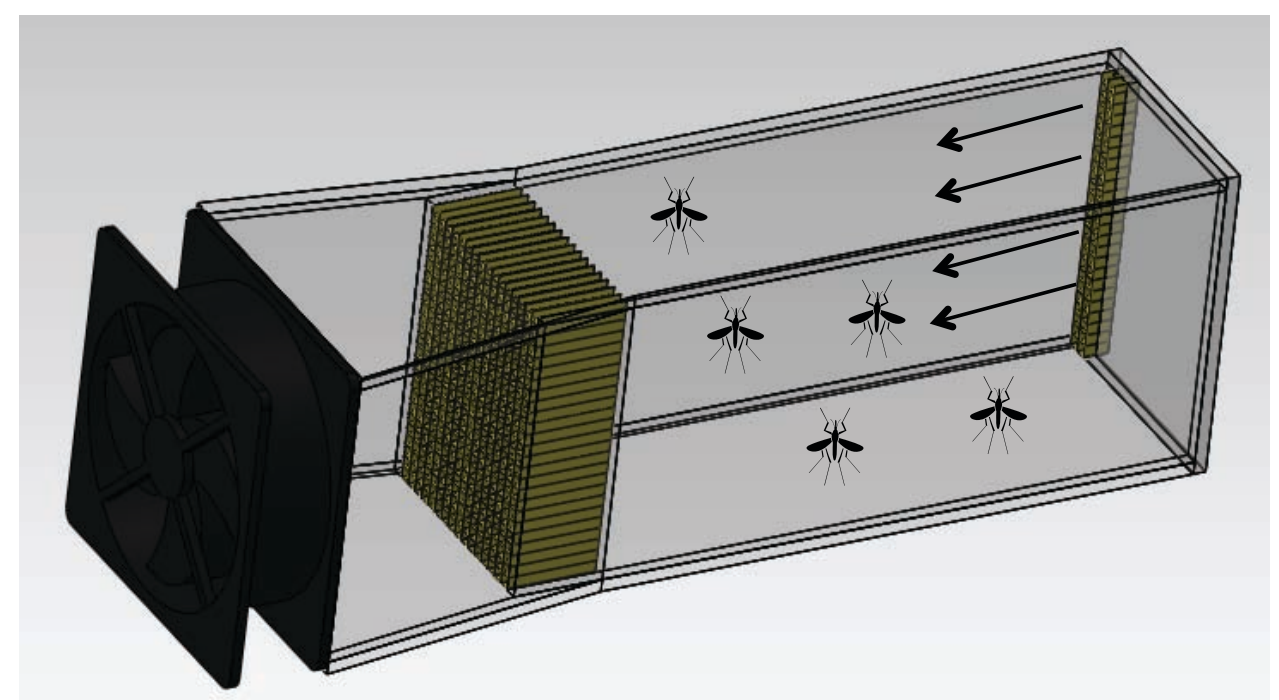

Figure 6: Miniature wind tunnel used to visualized mosquito flight upon entering a dense fog stream and takeoff. A variable speed computer fan pulls in fog at $3 \mathrm{~cm} / \mathrm{s}$ as denoted by the arrows. 